\title{
Evaluation of antioxidant capacity of Aidia borneensis leaf infusion, an endemic plant in Brunei Darussalam
}

\author{
${ }^{1}$ Metussin, N., ${ }^{2}$ Mohamed, H., ${ }^{2}$ Ahmad, N., ${ }^{1 *}$ Yasin, H.M. and ${ }^{1}$ Usman, A. \\ ${ }^{1}$ Department of Chemistry, Faculty of Science, Universiti Brunei Darussalam, Jalan Tungku Link, Gadong \\ BE1410, Negara Brunei Darussalam \\ ${ }^{2}$ Department of Biology, Faculty of Science, Universiti Brunei Darussalam, Jalan Tungku Link, Gadong \\ BE1410, Negara Brunei Darussalam
}

\section{Article history:}

Received: 10 June 2017

Received in revised form: 3

August 2017

Accepted: 4 August 2017

Available Online: 7 August

2017

Keywords:

Aidia borneensis,

Antioxidant,

Total phenolic content,

Total flavonoid content,

Total flavanol content

DOI:

https://doi.org/10.26656/fr.2017.2(1).109

\begin{abstract}
We investigated the total antioxidant capacity of Aidia borneensis leaf infusion, a Bornean endemic plant, which is traditionally consumed as a home-remedy beverage in Brunei Darussalam. The antioxidant capacity of the infusion of $A$. borneensis leaves was evaluated by 2,2-diphenyl-1-picryhydrazyl (DPPH) radical-scavenging ability. We found that the infusion shows a relatively high antioxidant capacity, and it was attributed to its high phenolic, flavonoid, and flavanol contents which were evaluated by Folin-Ciocalteu reagent, colorimetric assay, and aluminum chloride colorimetric method, respectively. By comparing its total antioxidant capacity, we estimated that the infusion of $A$. borneensis leaves is in the middle rank among twelve different commercially available Camellia sinensis teas. Our findings would have significant implications on $A$. borneensis products from Brunei Darussalam and on the feasibility of establishing this new beverage among the commercially available conventional $C$. sinensis and herbal teas.
\end{abstract}

\section{Introduction}

Antioxidants are compounds that inhibit the formations of free radicals and lipid peroxidations, which are responsible for many diseases and aging process in the human body (Ostrowska et al., 2001; Karimi et al., 2011; Gonbad et al., 2015). Among the antioxidants, polyphenol derivatives such as phenolic, flavonoid, and flavanol contents naturally found in plants are the most beneficial (Salah et al., 1995; Zandi and Gordon, 1995; Kim et al., 2003; Karimi et al., 2011). In addition to those polyphenols, the existences of phytochemical constituents, such as ascorbic acid, tocopherols, and carotenoids in consumable plants enhance their total antioxidant capacity (Chatterjee et al., 2013). Therefore, entire leaves either raw, cooked or processed into beverages are commonly consumed. Several research on herbal plants have suggested that their vitamins $\mathrm{A}, \mathrm{C}$, and $\mathrm{E}$, carotenoids, polyphenolics and flavonoid contents also have antioxidant capacities (Karimi et al., 2011). Thus, they have medicinal values in the treatment of many human diseases, including cancer, cardiovascular diseases, and inflammatory diseases (Krishnaiah et al., 2011). For instance, the herbal tea infusions (also known as tisanes) made from fresh or dried flowers, leaves, seeds or roots are also widely consumed worldwide. These herbal tea infusions are believed to have medicinal effects, especially for their stimulant, relaxant, or sedative properties (Kara, 2009).

In case of beverages, many studies have confirmed that leaves of tea (Camellia sinensis which belongs to Theaceae family) possess considerable antioxidant capacity (Yen and Chen, 1995; Robinson et al., 1997; Rice-Evans, 1999; Gardner et al., 2007; Pekal et al., 2012; Gonbad et al., 2015; Hajiaghaalipour et al., 2016). It has also been demonstrated that flavonoid compounds such as epigallocatechin gallate, epicatechin gallate, epigallocatechin, and epicatechin accumulated in tea leaves at all their fermentation levels act as effective scavengers of free radicals (Salah et al., 1995). More interestingly, the polyphenols extracted from tea infusions in ethanol solution have stronger antioxidant, anticarcinogenic, and antimutagenic properties, but they are less toxic as compared with the synthetic antioxidants such as butylated hydroxyanisole, butylated hydroxytoluene and dl- $\alpha$-tocopherol (Chen and Wan, 1994). Therefore, $C$. sinensis tea has been considered to 
have potential health-promoting properties, due to its ability to reduce the risks of coronary heart disease (Stensvold et al., 1992; Emerit, 1994; Halliwell, 1996; Hertog et al., 1997), stroke incidence (Keli et al., 1995; Peters et al, 2001), chronic inflammation (De Bacquer et al., 2006), and cancer incidence (Arab and Il'yasova, 2003; Chung et al, 2003).

Driven by such high health benefits of natural antioxidants, exploration on new potential plants possessing high antioxidant capacity is of interest (Ariffin et al., 2011). Through such exploration, one could expect that there will be more varieties of healthy and good quality beverages in addition to the existing $C$. sinensis teas. In this regard, we have focused our research efforts on plants locally grown in Brunei Darussalam, where endemic plant species are remarkably plentiful. Those plants are uncultivated, but many of them have been utilized in traditional medicines to treat many diseases. Several of those important medicinal plants have been reported by Goh et al. (2017), but extensive researches on those plants are currently pursued. One of those plants is Aidia borneensis, a Bornean endemic plant and locally known as Sambah bagangan, belonging to Rubiaceae family (Goh et al., 2017). In general, this family includes perennial evergreen plants, which require humid and warm environmental geographic regions. A. borneensis grows up to about $2 \mathrm{~m}$ high, as depicted in Figure 1A. This plant thrives well on hilly and drained soils under the shadow of big trees, and it has the unique pinkish-red color of young leaves and green color of matured leaves, as shown in Figure 1B-C.
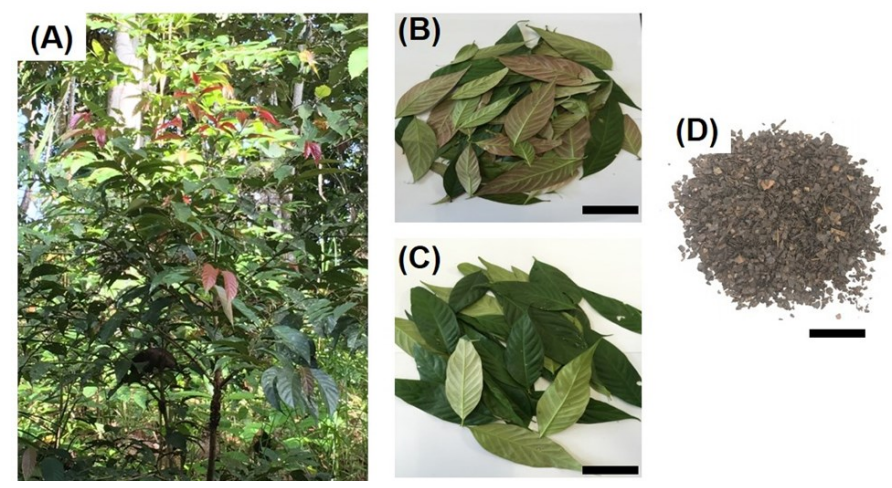

Figure 1. Representative images of (A) the plant, (B) young leaves, (C) matured leaves, and (D) the commercial tea of $A$. borneensis. The scale bars of $10 \mathrm{~cm}$ are shown in the images B-D.

Traditionally, infusion of $A$. borneensis leaves are treated as beverages, where a decoction is prepared from the crude or dried leaves. It is consumed as a hot drink and it is believed to be able to relieve body aches, tiredness, and gastric pains (Goh et al., 2017). Intensive researches characterizing and quantifying the beneficial constituents as well as principle substances in this particular plant leaves are indispensable. In the present study, we have evaluated the antioxidant capacity of infusion A. borneensis leaves (Figure 1D).

In order to assess relative potential of infusion of $A$. borneensis leaves, we have also compared its total antioxidant capacity to those found in twelve different commercially available $C$. sinensis black, $\mathrm{Pu} \mathrm{Er}$, and herbal teas. We should consider that this is a comparison without assessing numerous factors that may affect chemical constituents in the commercial teas according to cultivation type, growth conditions, horticultural practices, and different treatments by the tea manufacturer. The relationship between the total antioxidant capacity and total phenolic, flavonoid, flavanol, catechin, and caffeine contents in the infusion of $A$. borneensis leaves were also investigated. This is the first report on the antioxidant capacity and chemical constituents in $A$. borneensis leaf infusion. Our findings would have significant implications on $A$. borneensis tea and on its feasibility among the conventional commercially available $C$. sinensis and other herbal teas.

\section{Materials and methods}

\subsection{Chemicals and equipment}

Dried leaves or tea of $A$. borneensis was obtained from the local factory (3MPK Herbal Tea, Kampong Kiudang, Brunei Darussalam). All chemicals in this work were obtained from different sources; namely 2,2diphenyl-1-picrylhydrazyl radical (DPPH), gallic acid monohydrate, quercetin, and catechin hydrate were purchased from Sigma-Aldrich (St. Louis, MO, USA), Folin-Ciocalteu reagent, sodium carbonate, sodium nitrite, aluminium chloride, sodium acetate, and methanol were purchased from Merck (DarmstadtGermany), and caffeine were obtained from Fluka (Buchs, Switzerland). All these chemicals were mainly in analytical grade, except for catechin hydrate (in HPLC grade), and they were used as received without any further purifications. Distilled water was used throughout the sample preparations and measurements. All absorption measurements were performed on a singlebeam UV-visible spectrophotometer (Optizen model $1412 \mathrm{~V}$, Korea). In all measurements, the sample was contained in a $2 \mathrm{~mL}$ disposable cuvette with $1.2 \mathrm{~cm}$ optical path, and the absorbance was recorded at a specific wavelength.

\subsection{General procedure of sample preparations}


Infusion of $A$. borneensis leaves was prepared using an aqueous extraction according to a standard procedure, similarly to typical household tea preparation. Briefly, 2 $\mathrm{g}$ of granular dried leaves of $A$. borneensis (Figure 1D) was soaked in $200 \mathrm{~mL}$ distilled water while stirring thoroughly with a glass rod, allowing their constituents to infuse into the solution. The infusion was then passed through a filter paper (Whatman Grade 1) with the pore size of $11 \mu \mathrm{m}$, and the filtrate was kept at room temperature to cool down to $20-25^{\circ} \mathrm{C}$. For chemical analyses, depending on each specific assay the infusion was diluted with distilled water to achieve concentration of the substances roughly within 10-4000 ppm.

\subsection{The effects of brewing temperature and brewing time}

To evaluate the effect of brewing temperature and time on the antioxidant capacity, we have prepared an infusion by soaking $2 \mathrm{~g}$ of dried leaves of $A$. borneensis directly into $200 \mathrm{~mL}$ distilled water. For the effect of brewing temperature, the distilled water was set to be at $10,20,40,60,80$, and $100^{\circ} \mathrm{C}$ for a constant brewing time at $3 \mathrm{~min}$. On the other hand, the effect of brewing time was inspected from the samples prepared in the distilled water at $80^{\circ} \mathrm{C}$ with various brewing time in the range of 1-120 min.

\subsection{Determination of total antioxidant capacity by DPPH assay}

The total antioxidant activity of the infusion of $A$. borneensis leaves was determined using 2,2-diphenyl-1picrylhydrazyl (DPPH) free radical scavenging assay according to the modified procedures reported by Delgado-Andrade et al. (2005). Here, a $200 \mu \mathrm{L}$ of the infusion with various concentrations within 10-1000 ppm was added into freshly prepared $1 \mathrm{~mL}$ DPPH (50 ppm) in methanol. The mixture was allowed to stand in the dark at room temperature for $30 \mathrm{~min}$. The monochromatic absorption of the mixture then was recorded at $517 \mathrm{~nm}$ against methanol, and a DPPH solution without infusion was used as a blank or control solution. The DPPH free radicals in the solution are scavenged by an antioxidant or a free radical species, resulting in a decrease in the intensity of its absorption band. Consequently, lower absorption intensity of the mixture indicated higher free radical scavenging activity (Yen et al., 1997), and DPPH free radical scavenging activities of the infusions were calculated according to

$\%$ inhibition of DPPH activity $=\left[\frac{A_{\text {control }}-A_{\text {sample }}}{A_{\text {control }}}\right] \times 100 \%$ where $A_{\text {control }}$ is the absorbance of the control solution and $A_{\text {sample }}$ is the absorbance of the infusion. We have plotted a curve of \% inhibition of DPPH activity against concentration of the infusions (data not shown), and from the plot we deduced $\mathrm{IC}_{50}$ value which was attributed to the concentration of the infusions required for $50 \%$ inhibition. Radical scavenging activities of wellknown antioxidants, namely gallic acid monohydrate and quercetin, have also been measured for comparison or calibration. Thus, the total antioxidant activity of the infusions associated with that of gallic acid calibration curve is considered as gallic acid equivalents (GAE) and it is expressed as in ppm standard DPPH equivalent.

\subsection{Determination of total phenolic content}

Total phenolic content (TPC) in the infusions was determined according to Folin-Ciocalteu method based on the procedure used by Chan et al. (2007). For this TPC determination, $300 \mu \mathrm{L}$ of diluted infusions were introduced into sample vial followed by $1.2 \mathrm{~mL}$ sodium carbonate $(7.5 \% \mathrm{w} / \mathrm{v})$ and $1.5 \mathrm{~mL}$ Folin-Ciocalteu reagent (diluted by 10 fold) in distilled water. The color of the mixture turned from yellow to blue, and this mixture was allowed to stand for $30 \mathrm{~min}$ at room temperature. Absorption of the mixture then was recorded at a single wavelength at $765 \mathrm{~nm}$. The calibration curve of gallic acid was plotted in the range of 25-200 ppm. The TPC of the infusion was calculated from linear regression of the calibration curve. Thus, TPC was expressed as gallic acid equivalents (GAE) in $\mathrm{mg} / 100 \mathrm{~g}$ dry sample.

\subsection{Determination of total flavonoid content}

Total flavonoid content (TFC) in the infusion was measured using a slightly modified colorimetric assay according to the procedure reported by Damiani et al. (2014). Briefly, $100 \mu \mathrm{L}$ of the infusions was dissolved in $1.35 \mathrm{~mL}$ distilled water in a sample vial. Into the solution, $50 \mu \mathrm{L}$ sodium nitrite $(5 \% \mathrm{w} / \mathrm{v})$ were added, followed by $50 \mu \mathrm{L}$ aluminium chloride $(10 \% \mathrm{w} / \mathrm{v})$. The mixture was mixed well using vortex (Model V-1 plus, Biosan, Latvia) and it was allowed to stand at room temperature in the dark for $10 \mathrm{~min}$. Similarly, we have appropriately prepared standard ethanolic solutions of catechin hydrate in the same way. Absorption of the mixture and the standard solutions was recorded at a single wavelength at $415 \mathrm{~nm}$. The TFC of the infusion was determined from linear regression of a calibration curve, which was obtained from catechin hydrate with different concentrations in the range of $25-1000 \mathrm{ppm}$. 
Therefore, the TFC of the infusion was expressed as catechin equivalent in $\mathrm{mg} / 100 \mathrm{~g}$ dry sample.

\subsection{Determination of total flavanol content}

Total flavanol (TF) content in the infusions was determined using the aluminium chloride colorimetric method according to the procedure reported by Iqbal et al. (2015) with slight modifications. Briefly, the infusion $(1.5 \mathrm{~mL})$ was put in a test tube, followed by addition of 1 $\mathrm{mL}$ aluminium chloride $(2 \% \mathrm{w} / \mathrm{v})$ in distilled water. Then, $3 \mathrm{~mL}$ sodium acetate $(5 \% \mathrm{w} / \mathrm{v})$ was added, and the mixture was mixed well using vortex. The mixture then was centrifuged at $3000 \mathrm{rpm}$ for $20 \mathrm{~min}$. The absorbance of the supernatant was measured at $440 \mathrm{~nm}$. In this measurement, ethanolic quercetin solutions which were prepared similarly to the samples of the infusions were used as a standard, and the calibration curve was obtained from the absorbance of the standard solutions with different concentrations in the range of 1-100 ppm. The TF of the infusion was determined from linear regression of the calibration curve, and it is expressed as quercetin equivalent concentration in $\mathrm{mg} / 100 \mathrm{~g}$ dry sample.

\subsection{Data analysis}

In this work, appropriate treatments were carried out throughout all the experiments. All measurements on the infusions and control solutions have been performed at least in triplicate and all data have been analyzed. The data presented in this report represent the average values.

\section{Results and discussion}

\subsection{Total antioxidant capacity of A. borneensis leaf infusion}

The total antioxidant capacity of the infusion of $A$. borneensis leaves has been analyzed by quantifying their $\mathrm{DPPH}$ free radical scavenging. The $\mathrm{IC}_{50}$ of $2 \mathrm{~g}$ dried leaves of A. borneensis infused in $200 \mathrm{ml}$ water was measured to be within 312 to $805 \mathrm{ppm}$, equivalent to 1440 to $3730 \mathrm{mg}$ gallic acid per $100 \mathrm{~g}$ dry sample of $A$. borneensis leaves. This indicates that the infusion of $A$. borneensis leaves is a significant source of antioxidants. According to the DPPH free radical scavenging, the total antioxidant capacity depends on the brewing time and temperature of the infusions, as described below.

\subsection{Effect of brewing time}

By plotting $\mathrm{IC}_{50}$ of the infusion against brewing time, as shown in Figure 2A, we demonstrate that its total antioxidant capacity increases gradually with the

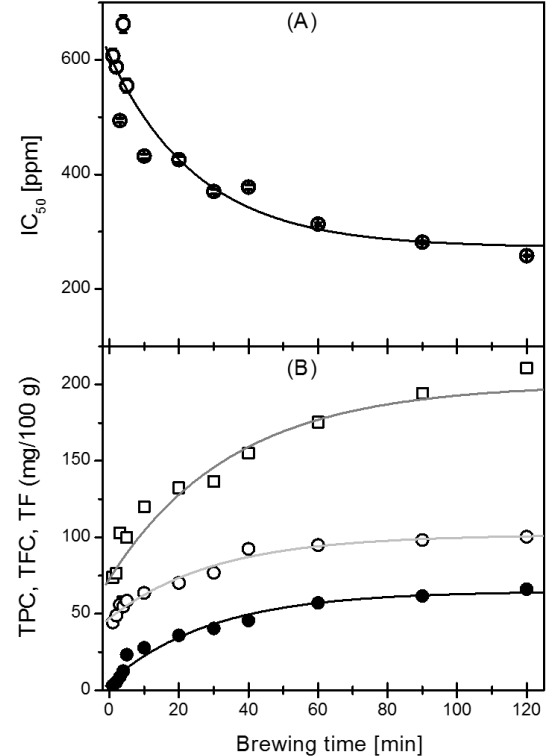

Figure 2. The effect of different infusion time on; (A) the total antioxidant activity, and (B) on the TPC (口), TFC (O), and TF $(\bullet)$ contents of $A$. borneensis tea. The solid lines are the best single exponential fits for the data. In the plot, the TPC has been scaled down by factor of 10 for the sake of clarity.

brewing time. The similar trend has also been observed in all C. sinensis teas (Ryan and Petit, 2010; Fernando and Soysa, 2015; Nikniaz et al., 2016). This may not be surprising because there are more phytochemicals and polyphenol derivatives could be permeated from the leaves into the solution at longer infusion time. However, we should note that at brewing temperature, $80^{\circ} \mathrm{C}$, more than $50 \%$ of the total antioxidant capacity was achieved during the first $3 \mathrm{~min}$ infusion time. The total antioxidant capacity increases exponentially with infusion time and it is saturated at infusion times longer than 1 hour, indicating that the optimum extraction capacity is reached. This implies that water could effectively extract the antioxidants from $A$. borneensis leaves, and most of them should pass through the openings or interstices on the leaves in the early time of infusion. We may consider that this phenomenon should be similar to the cases of a liquid passing through a membrane due to different concentrations, where its concentration increases exponentially. Therefore, the concentrations of the antioxidants in the infusion may obey first- or secondorder kinetics (Peleg, 1988; Lafka et al., 2013; Fernando and Soysa, 2015). To clarify this issue, we have fitted our data with a single exponential function. As shown in Figure 2A, the single-exponential fitting gives an infusion rate constant of the antioxidants to be $0.040 \pm 0.005 \mathrm{~min}^{-1}$. The validity of this consideration is unambiguously supported by the infusion-time dependence of TPC, TFC, and TF content of $A$. borneensis leaves, where their infusion rate constant is also within the range of $0.035-0.040 \mathrm{~min}^{-1}$, as shown in 
Figure 2B. These findings further indicate that the total antioxidant of $A$. borneensis leaves is clearly related to its TPC, TFC, and TF contents. We may also note that TPC, TFC, and TF contents, which are considered to be an important criterion of tea quality, were measured to be in the range of 739-2110, 440-1005, and 3.0-66.5 mg per $100 \mathrm{~g}$ dried leaves of $A$. borneensis, respectively.

\subsection{Effect of brewing temperature}

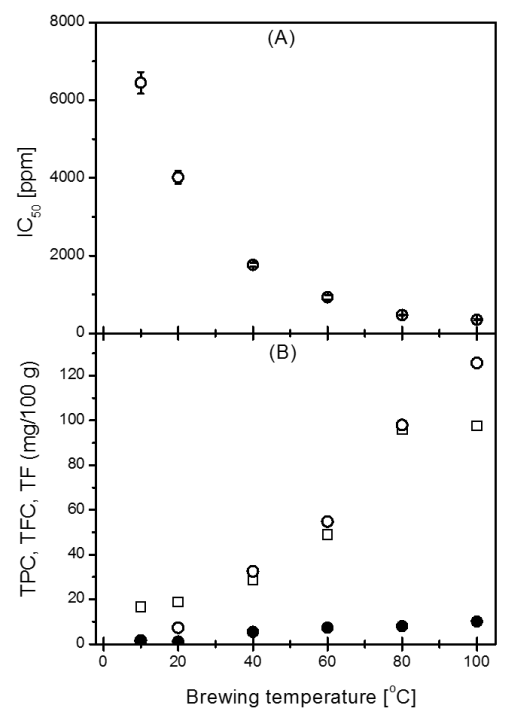

Figure 3. The effect of different infusion temperature on; (A) the total antioxidant activity, and (B) on the TPC ( $\square$ ), TFC $(\circ)$, and TF $(\bullet)$ contents of $A$. borneensis tea (B). In the plot, the TPC has been scaled down by factor of 10 for the sake of clarity.

Figure 3A shows that the total antioxidant capacity of $A$. borneensis leaves increases abruptly with brewing temperature (in the range of $10-100^{\circ} \mathrm{C}$ ). A similar tendency has also been observed for $C$. sinensis black and green teas, where infusion with greater antioxidant capacity was obtained at longer brewing time and higher temperatures (Sharpe et al., 2016). This finding implies that more phytochemicals and polyphenol derivatives from the $A$. borneensis leaves are infused into the water due most probably to the existences of more openings and interstices on the leaves, increasing the diffusion rate at higher brewing temperature. We found that more than $90 \%$ of the total antioxidant capacity was achieved at temperature higher than $60^{\circ} \mathrm{C}$, indicating efficient releases of the phytochemicals and polyphenol derivatives from $A$. borneensis leaves into the brewing solution due most probably to larger numbers of openings, interstices, denaturation, or disruption of their cells at temperature higher than $60^{\circ} \mathrm{C}$.

Accordingly, in Figure 3B, we show that TPC, TFC, and $\mathrm{TF}$ contents in the infusion of $A$. borneensis leaves also increase abruptly with brewing temperature.
Therefore, we could draw a conclusion that the total antioxidant capacity is related to the concentrations of phytochemicals and polyphenol derivatives in the $A$. borneensis leaf infusion, as discussed above. Considering that TF content in the infusions to scavenge $\mathrm{DPPH}$ is one or two orders lower when compared with those of TPC and TFC (see Figures $2 \mathrm{~B}$ and $3 \mathrm{~B}$ ), thus polyphenols and flavonoids should be the major constituents responsible for the total antioxidant capacity of $A$. borneensis leaves.

It is noteworthy that although caffeine does not belong to polyphenols, it has a capability to inhibit lipid peroxidation (Brezova et al., 2009). Thus, one may consider that caffeine content could have a contribution in the antioxidant capacity of $A$. borneensis leaves. In this sense, we have measured its caffeine content, and we found that it is negligibly small (data not shown). Therefore, the contribution of caffeine in the total antioxidant capacity of $A$. borneensis leaves is not important. Such negligible contribution of caffeine in the total antioxidant capacity has also been discussed for $C$. sinensis black tea infusions (Fernando and Soysa, 2009).

\subsection{Antioxidant capacity of several commercially available teas}

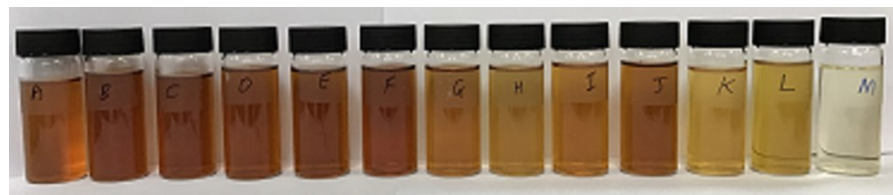

Figure 4. The images of aqueous infusions of commercially available $A$. borneensis of $3 \mathrm{MPK}$ (I) and $C$. sinensis black teas of various brands (A, B, C, D, E, F, G, H, J, K, L, and M) brewed for $3 \mathrm{~min}$ at $80^{\circ} \mathrm{C}$.

We should note that most of the health benefits of tea are based on its total antioxidant activity. The total antioxidant activity of the infusion of $A$. borneensis leaves, therefore, was compared with those of twelve different $C$. sinensis black, herbal, and $\mathrm{Pu} \mathrm{Er}$ teas commercially available. With the same brewing time and temperature, color of the infusions clearly varies depending on their brands, as shown in Figure 4. We found that the total antioxidant activity, either in $\mathrm{IC}_{50}$, gallic acid equivalent antioxidant capacity (GEAC), or quercetin equivalent antioxidant capacity (QEAC), is also significantly different in the various brands of teas. As listed in Table 1, the minimum $\mathrm{IC}_{50}(143 \mathrm{ppm})$ was observed in the tea infusion of brand $\mathbf{A}$, while the maximum value (4078 ppm) was found in the infusion of brand M. This indicates that the total antioxidant capacity of black tea brand $\mathbf{A}$ is approximately 30 -fold higher than that of brand $\mathbf{M}$. We should note that 
infusion of $A$. borneensis leaves (brand $\mathbf{I}$ ) has an $\mathrm{IC}_{50}$ of $592 \mathrm{ppm}$, equivalent to approximately 4-fold lower than that of brand $\mathbf{A}$ and it is 7-fold higher than of brand $\mathbf{M}$. This means that the health benefits of $A$. borneensis infusion are in the middle rank among the twelve $C$. sinensis and herbal teas evaluated in the present study.

Table 1. Antioxidant activity of aqueous infusions of commercially available $A$. borneensis and $C$. sinensis black teas brewed for 3 min at $80^{\circ} \mathrm{C}$.

\begin{tabular}{cccc}
\hline Tea brand & $\mathrm{IC}_{50}$ & QEAC & GEAC \\
\hline $\mathrm{A}$ & $143 \pm 7$ & $11097 \pm 611$ & $8179 \pm 450$ \\
$\mathrm{~B}$ & $222 \pm 14$ & $7140 \pm 440$ & $5262 \pm 324$ \\
$\mathrm{C}$ & $248 \pm 2$ & $6377 \pm 63$ & $4700 \pm 47$ \\
$\mathrm{D}$ & $265 \pm 14$ & $5985 \pm 303$ & $4412 \pm 223$ \\
$\mathrm{E}$ & $271 \pm 10$ & $5831 \pm 221$ & $4298 \pm 163$ \\
$\mathrm{~F}$ & $297 \pm 19$ & $5335 \pm 345$ & $3932 \pm 254$ \\
$\mathrm{G}$ & $410 \pm 22$ & $3860 \pm 212$ & $2845 \pm 156$ \\
$\mathrm{H}$ & $535 \pm 39$ & $2967 \pm 223$ & $2187 \pm 164$ \\
$\mathrm{I}$ & $592 \pm 27$ & $2675 \pm 122$ & $1972 \pm 90$ \\
$\mathrm{~J}$ & $641 \pm 32$ & $2471 \pm 127$ & $1821 \pm 94$ \\
$\mathrm{~K}$ & $673 \pm 41$ & $2353 \pm 140$ & $1735 \pm 103$ \\
$\mathrm{~L}$ & $3364 \pm 152$ & $470 \pm 21$ & $347 \pm 15$ \\
$\mathrm{M}$ & $4078 \pm 42$ & $388 \pm 4$ & $286 \pm 3$ \\
\hline
\end{tabular}

We should note that, in addition to the brewing conditions (brewing time and temperature), the total antioxidant capacity of teas should vary greatly depending on a wide variety of factors such as the location of plantation, harvesting season, drying method, and manufacturing. Although there have been several literatures comparing the total antioxidant capacity and polyphenolic contents of commercial teas from different various brands, those factors are simply neglected (Chan et al., 2007; Taheri et al., 2011; Carloni et al., 2013). In particular, black teas are produced by fermentation with a number of parameters which can also modify their antioxidant capacity. For instance, polyphenol oxidases released from the disrupted cells can react with catechins, thus during black tea manufacturing, the most considerable changes occur in black teas is the concentration of catechins. Therefore, it is obvious that there is a significant difference in the total antioxidant capacity found in commercial black teas from various brands. However, we should mention that, regardless of that wide variety of factors determining tea quality, the infusion of $A$. borneensis leaves which was processed traditionally is among those teas with considerable total antioxidant capacity. In order to explore the effect of processing, a study on the antioxidant capacity of $A$. borneensis leaves with different pretreatments is currently being pursued.

\section{Conclusion}

In summary, we have investigated the total antioxidant capacity of Aidia borneensis leaf infusion, a Bornean endemic plant, by measuring its DPPH free radical scavenging activity. We found that the antioxidant capacity of the infusion increases with brewing time and temperature, and we have attributed the total antioxidant capacity of the infusion to their TPC, TFC, and TF contents. We have also assessed relative potential of the infusion of $A$. borneensis leaves by comparing its total antioxidant capacity with twelve different $C$. sinensis black, herbal, and $\mathrm{Pu}$ Er teas commercially available. We found that the infusion of $A$. borneensis leaves is in the middle rank among the twelve commercially available teas. Therefore, the present preliminary study has highlighted significant implications on A. borneensis leaves and on the feasibility of establishing this new potential plant among commercially available conventional $C$. sinensis tea, which has been known as a rich source of antioxidants.

\section{Acknowledgements}

This project was financially supported by Universiti Brunei Darussalam under the research grant BRC/6. The authors would like to thank $\mathrm{Hj}$ Amran $\mathrm{Hj}$ Johari (Deputy Chairman of the 3MPK Tea Factory) and $\mathrm{Hj}$ Mohd Saiful bin Abdullah Anja (the Village Head of Kampong Kiudang, Tutong, Brunei Darussalam) for providing us fresh samples of $A$. borneensis leaves and plants. The authors also thank Dr. J.W. Ferry Slik for helping us to identify the plant species. The authors declare that they have no conflict of interest.

\section{References}

Arab, L. and Il'yasova, D. (2003). The epidemiology of tea consumption and colorectal cancer incidence. Journal of Nutrition, 133, 3310S-3318S.

Ariffin, F., Chew, S.H., Bhupinder, K., Karim, A.A. and Huda, N. (2011). Antioxidant capacity and phenolic composition of fermented Centella asiatica herbal teas. Journal of Science Food Agriculture, 91, 27312739.

Brezova, V., Šlebodova, A. and Staško, A.A. (2009). Coffee as a source of antioxidants: An EPR study. Food Chemistry, 114, 859-868. 
Carloni, P., Tiano, L., Padella, L., Bacchetti, T., Chisomo Customu, C., Kay, A. and Damiani, E. (2013). Antioxidant activity of white, green and black tea obtained from the same tea cultivar. Food Research International, 53, 900-908.

Chan, E.W.C., Lim, Y.Y. and Chew, Y.L. (2007). Antioxidant activity of Camellia sinensis leaves and tea from a lowland plantation in Malaysia, Food Chemistry, 102, 1214-1222.

Chatterjee, S., Chatterjee, S., Dey, K.K. and Dutta, S. (2013). Study of antioxidant activity and immune stimulating potency of the ethnomedicinal plant, Cassia alata (L.) Roxb. Medicinal Aromatic Plants, 2, 1-6.

Chen, W.J. and Wan, S.Q. (1994). Research progress on polyphenols of tea. Natural Product Research and Development, 6, 74-80.

Chung, F.L., Schwarta, J., Herzog, C.R. and Yang, Y.M. (2003). Tea and cancer prevention: studies in animals and humans. Journal of Nutrition, 133, 3268S-3274S.

Damiani, E., Bacchetti, T., Padella, L., Tiano, L. and Carloni, P. (2014). Antioxidant activity of different white teas: Comparison of hot and cold tea infusions. Journal of Food Compositional Analysis, 33, 59-66.

De Bacquer, D., Clays, E., Delanghe, J. and De Backer, G. (2006). Epidemiological evidence for an association between habitual tea consumption and markers of chronic inflammation. Atherosclerosis, 189, 428-435.

Delgado-Andrade, C., Rufiaan-Henares, J.A. and Morales, F.J. (2005). Assessing the antioxidant activity of melanoidins from coffee brews by different antioxidant methods. Journal of Agricultural Food Chemistry, 53, 7832-7836.

Emerit, I. (1994). Reactive oxygen species, chromosome mutation and cancer: possible role of clastogenic factors in carcinogenesis. Free Radical Biological Medicine, 16, 99-109.

Fernando, C.D. and Soysa, P. (2015). Extraction Kinetics of phytochemicals and antioxidant activity during black tea (Camellia sinensis L.) brewing. Nutritional Journal, 14, 74.

Gardner, E.J., Ruxton, C.H.S. and Leeds, A.R. (2007). Black tea-helpful or harmful? A review of the evidence. European Journal of Clinical Nutrition, 61, 3-18.

Goh, M.P.Y., Basri, A.M., Yasin, H.M., Taha, H. and Ahmad, N. (2017). Ethnobotanical review and pharmacological properties of selected medicinal plants in Brunei Darussalam: Litsea elliptica, Dillenia suffruticosa, Dillenia excelsa, Aidia racemosa, Vitex pinnata and Senna alata. Asian Pacific Journal of Tropical Biomedicine, 7, 173-180.

Gonbad, R.A., Afzan, A., Karimi, E., Sinniah, U.R. and Swamy, M.K. (2015). Phytoconstituents and antioxidant properties among commercial tea (Camellia sinensis L.) clones of Iran. Electronic Journal of Biotechnology, 18, 433-438.

Hajiaghaalipour, F., Sanusi, J. and Kanthimathi, M.S. (2016). Temperature and time of steeping affect the antioxidant properties of white, green, and black tea. Journal of Food Science, 81, H246-H254.

Halliwell, B. (1996). Oxidative stress, nutrition and health. Experimental strategies for optimization of nutritional antioxidant intake in human. Free Radical Research, 25, 57-74.

Hertog, M.G., Feskens, E.J. and Kromhout, D. (1997). Antioxidant flavonols and coronary heart disease risk. The Lancet, 349, 699.

Iqbal, E., Salim, K.A. and Lim L.B.L. (2015). Phytochemical screening, total phenolics and antioxidant activities of bark and leaf extracts of Goniothalamus velutinus (Airy Shaw) from Brunei Darussalam. Journal of King Saud UniversityScience, 27, 224-232.

Kara, D. (2009). Evaluation of trace metal concentrations in some herbs and herbal teas by principal component analysis. Food Chemistry, 114, 347-354.

Karimi, E., Jaafar, H.Z.E. and Ahmad, S. (2011). Phenolics and flavonoids profiling and antioxidant activity of three varieties of Malaysian indigenous medicinal herb Labisia pumila Benth. Journal of Medicinal Plant Research, 5, 1200-1206.

Keli, S.O., Hertog, M.G., Feskens, E.J. and Kromhout, D. (1995). Flavonoids, antioxidant vitamins and risk of stroke: the Zutphen Study. Archives Internal Medicines, 154, 637-642.

Kim, D.O., Jeong, S.W. and Lee, C.Y. (2003). Antioxidant capacity of phenolic phytochemicals from various cultivars of plums. Food Chemistry, 81, 321-326.

Krishnaiah, D., Sarbatly, R. and Nithyanandam, R. (2011). A review of the antioxidant potential of medicinal plant species. Food Bioproduct Processes, 89, 217-233.

Lafka, T.I., Lazou, A.E., Sinanoglou, V.J. and Lazos, E.S. (2013). Phenolic extracts from wild olive leaves 
and their potential as edible oils antioxidants. Foods, 2, 18-31.

Nikniaz, Z., Mahdavi, R., Ghaemmaghami, S.J., Yagin, N.L. and Nikniaz, L. (2016). Effect of different brewing times on antioxidant activity and polyphenol content of loosely packed and bagged black teas (Camellia sinensis L.). Avicenna Journal of Phytomedicine, 6, 313-321.

Ostrowska, J., Stankiewicz, A. and Skrzydlewska, E. (2001). Antioxidative properties of green tea. Bromatology and Toxicology Chemistry, 2, 131.

Pekal, A., Drózdz, P., Biesaga, M. and Pyrzynka, K. (2012). Screening of the antioxidant properties and polyphenol composition of aromatised green tea infusions. Journal of Science Food Agriculture, 92, 2244-2249.

Peleg, M. (1988). An empirical model for the description of moisture sorption curves. Journal of Food Science, 53, 1216-1217.

Peters, U., Poole, C. and Arab, L. (2001). Does tea affect cardiovascular disease? A meta analysis. American Journal of Epidemiology, 154, 495-503.

Rice-Evans, C. (1999). Implications of the mechanisms of action of the tea polyphenols as antioxidants in vitro for chemoprevention in humans. Proceeding of Society Experimental Biology and Medicine, 220, 262-266.

Robinson, E.E., Maxwell, S.R.J. and Thorpe, G.H.G. (1997). An investigation of the antioxidant capacity of black tea using enhanced chemiluminescence. Free Radical Research, 26, 291-302.

Ryan, L. and Petit, S. (2010). Addition of whole, semi skimmed, and skimmed bovine milk reduces the total antioxidant capacity of black tea. Nutritional Research, 30, 14-20.

Salah, N., Miller, N.J., Parganga, G., Tifburg, L., Bolwell, G.P. and Rice-Evans, C. (1995). Polyphenolic flavanols as scavengers of aqueous phase radicals and as chain breaking antioxidant. Archives of Biochemistry and Biophysics, 32, 339346.

Sharpe, E., Hua, F., Schuckers, S., Andreescu, S. and Bradley, R. (2016). Effects of brewing conditions on the antioxidant capacity of twenty-four commercial green tea varieties. Food Chemistry, 192, 380-387.

Stensvold, I., Tverdal, A., Solvoll, K. and Foss, O.P. (1992). Tea consumption. Relationship to cholesterol, blood pressure, and coronary and total mortality. Preventive Medicine, 21, 546-553.

Taheri, M., Giahi, M., Shahmohamadi, R., Ghafoori, H.,
Aghamaali, M.R. and Sariri, R. (2011). Screening antioxidant activity of extracts from different tea samples. Pharmacologyonline, 3, 442-448.

Yen, G.C. and Chen, H.Y. (1995). Antioxidant activity of various tea extracts in relation to their antimutagenicity. Journal of Agricultural Food Chemistry, 44, 701-705.

Yen, G.C., Chen, H.Y. and Peng, H.H. (1997). Antioxidant and pro-oxidant effects of various tea extracts. Journal of Agricultural Food Chemistry, $45,30-34$.

Zandi, P. and Gordon, M.H. (1995). Stabilization of rapeseed oil to green tea extracts. In Proceeding of the international tea symposium, p. 216-227. Shanghai, China. 